\title{
Lipiodol Deposition and Washout in Primary and Metastatic Liver Tumors After Chemoembolization
}

\author{
NARIMAN NEZAMI ${ }^{1,2}$, JOHANNA MARIA MIJNTJE VAN BREUGEL ${ }^{3,4,5}$, MENELAOS KONSTANTINIDIS ${ }^{6}$, \\ JULIUS CHAPIRO ${ }^{1,3}$, LYNN JEANETTE SAVIC ${ }^{3,7,8}$, MILENA ANNA MISZCZUK ${ }^{3}$, IRVIN REXHA ${ }^{3,7}$, \\ MINGDE LIN ${ }^{9}$, KELVIN HONG $^{10}$ and CHRISTOS GEORGIADES ${ }^{10}$ \\ ${ }^{1}$ Section of Interventional Radiology, Department of Radiology and Biomedical Imaging, \\ Yale School of Medicine, New Haven, CT, U.S.A.; \\ ${ }^{2}$ Division of Vascular and Interventional Radiology, Department of Diagnostic Radiology and Nuclear Medicine, \\ University of Maryland School of Medicine, Baltimore, MD, U.S.A.; \\ ${ }^{3}$ Department of Radiology and Biomedical Imaging, Yale School of Medicine, New Haven, CT, U.S.A.; \\ ${ }^{4}$ Department of Radiology, University Medical Center Utrecht, Utrecht, the Netherlands; \\ ${ }^{5}$ Medical faculty, Utrecht University, Utrecht, the Netherlands; \\ ${ }^{6}$ Division of Biostatistics, Dalla Lana School of Public Health, University of Toronto, Toronto, ON, Canada; \\ ${ }^{7}$ Department of Radiology, Charité - Universitätsmedizin Berlin, \\ Corporate Member of Freie Universität Berlin and Humboldt-Universität zu Berlin, Berlin, Germany; \\ ${ }^{8}$ Berlin Institute of Health, Charité - Universitätsmedizin Berlin, Berlin, Germany; \\ ${ }^{9}$ Visage Imaging, Inc., San Diego, CA, U.S.A., \\ ${ }^{10}$ Division of Vascular and Interventional Radiology, Russel H. Morgan Department of Radiology \\ and Radiological Sciences, Johns Hopkins University School of Medicine, Baltimore, MD, U.S.A.
}

\begin{abstract}
Background/Aim: Lipiodol is the key component of conventional trans-arterial chemoembolization. Our aim was to evaluate lipiodol deposition and washout rate after conventional trans-arterial chemoembolization in intrahepatic cholangiocarcinoma and hepatic metastases originating from neuroendocrine tumors and colorectal carcinoma. Patients and Methods: This was a retrospective analysis of 44 patients with intrahepatic cholangiocarcinoma and liver metastasis from neuroendocrine tumors or colorectal carcinoma who underwent conventional trans-arterial chemoembolization. Lipiodol volume $\left(\mathrm{cm}^{3}\right)$ was analyzed on non-contrast computed tomography imaging obtained within 24 h post conventional trans-arterial chemoembolization, and 40-220 days after conventional trans-arterial chemoembolization using volumetric image analysis software. Tumor response was assessed on contrast-enhanced magnetic resonance imaging 1 month after conventional trans-arterial chemoembolization.
\end{abstract}

This article is freely accessible online.

Correspondence to: Nariman Nezami, MD, 22 S. Greene Street N2E23, Baltimore MD 21201, U.S.A. Tel: +1 4103696458, e-mail: dr.nezami@gmail.com

Key Words: Lipiodol, chemoembolization, deposition, intrahepatic cholangiocarcinoma, neuroendocrine tumors, colorectal carcinoma.
Results: The washout rate was longer for neuroendocrine tumors compared to colorectal carcinoma, with half-lives of 54.61 days $(p<0.00001)$ and 19.39 days $(p<0.001)$, respectively, with no exponential washout among intrahepatic cholangiocarcinomas $(p=0.83)$. The half-life for lipiodol washout was longer in tumors larger than $300 \mathrm{~cm}^{3}$ compared to smaller tumors (25.43 vs. 22.71 days). Lipiodol wash out half-life was 54.76 days $(p<0.01)$ and 29.45 days $(p<0.00001)$ for tumors with a contrast enhancement burden of $60 \%$ or more and less than $60 \%$, respectively. A negative exponential relationship for lipiodol washout was observed in nonresponders $(p<0.00001)$. Conclusion: Lipiodol washout is a time-dependent process, and occurs faster in colorectal carcinoma tumors, tumors smaller than $300 \mathrm{~cm}^{3}$, tumors with baseline contrast enhancement burden of less than $60 \%$, and non-responding target lesions.

Lipiodol is the key component of conventional trans-arterial chemoembolization (cTACE) for primary and metastatic liver tumors $(1,2)$. This agent serves as a tumor-seeking, embolic and drug carrier agent, and is used in combination with chemotherapy for cTACE (3). In addition, the radiopacity of lipiodol helps to easily visualize and track it $(4,5)$. It has been shown that lipiodol retention in the treated tumor is related to tumor necrosis (6). Therefore, it could be considered as an imaging surrogate for response (7). While there are different one-dimensional (1D), two-dimensional 


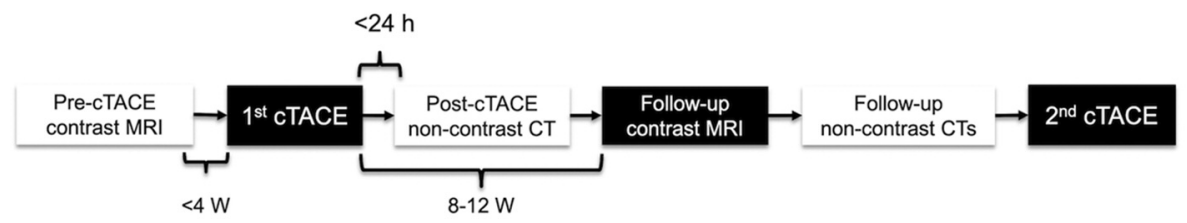

Figure 1. Treatment and Imaging sequence. This timeline illustrates the sequence of imaging and treatment with conventional trans-arterial chemoembolization (cTACE) was performed. CT: Computed tomography.

(2D), and three-dimensional (3D) methods to measure tumor response and lipiodol deposition, 3D or volumetric methods are more accurate for measuring lipiodol deposition within the targeted tumor (8).

Quantitative 3D metrics for tumor enhancement according to the European Association for the Study of the Liver (qEASL) have been introduced for evaluation and prediction of tumor response and survival (9), and have been shown to be better than 1D and 2D metrics in various tumor types, including neuroendocrine (NET), colorectal carcinoma (CRC) and hepatocellular carcinoma (HCC) (10-14). The qEASL metrics can be used for measurements of lipiodol deposition $(15,16)$. Our team previously showed that lipiodol deposition is higher in patients with HCC who responded to cTACE on both conventional and cone-beam computed tomography (CT) $(8,11)$. The degree of lipiodol deposition after cTACE in patients with NET was recently shown to predict response (17), although it is still unknown if lipiodol deposition is also a predictor of response in hepatic cholangiocarcinoma and liver metastases from colorectal tumors. Moreover, the rate of lipiodol washout (beyond absolute lipiodol uptake) has not been rigorously investigated as a potential indicator of response (18). A relationship between the rate of lipiodol washout and tumor response was first shown in a VX2 rabbit model of HCC (3). Tumor cells that remained viable after TACE showed washout of lipiodol through lysosomes, whereas dead tumor cells showed intra-cellular retention of small lipiodol droplets.

The objective of this study was to quantitatively measure lipiodol deposition and its washout rate after cTACE in patients with intrahepatic cholangiocarcinoma (ICC), hepatic metastasis from NET, and CRC.

\section{Patients and Methods}

Study design. This retrospective analysis of cases enrolled patients from three different clinical trials currently running at Yale New Haven Hospital (NCT02753881, NCT01877187, and NCT02994 251). These trials are Institutional Review Board-approved, and the retrospective data review required for this study was compliant with the Institutional policy.

Study population. All patients were reviewed in our multidisciplinary Liver Tumor Board and referred for cTACE.
Inclusion criteria were: i) Intrahepatic cholangiocarcinoma or hepatic metastases from NET or CRC treated with cTACE; ii). noncontrast CT scan within $24 \mathrm{~h}$ after cTACE; iii). at least one followup non-contrast CT scan; iv). multiphasic contrast-enhanced MRI study 8 to 12 weeks after the first cTACE. Figure 1 demonstrates the study flow chart and sequence of CT and MRI studies in relation to cTACE.

Conventional trans-arterial chemoembolization protocol. All treatments were cTACE procedures performed by one of two interventional radiologists (K.H. and C.G.), each with more than 15 years of experience in intra-arterial hepatic interventions. According to our institutional standard protocol (1), cTACE was performed using an approximately 1-to-1 mixture of $10 \mathrm{cc}$ lipiodol (Lipiodol; Guerbet, France) and water-soluble chemotherapy cocktail. The latter included $50 \mathrm{mg}$ doxorubicin (Adriamycin; Pharmacia \& Upjohn, Peapack, NJ, USA) and $10 \mathrm{mg}$ mitomycin C (Intas Pharmaceuticals Limited, Pharmez, Ahmedabad, India). All cTACE procedures were followed by administration of Embospheres (diameter 100-300 $\mu \mathrm{m}$; Merit Medical Co., South Jordan, UT, USA) to near stasis. The angiographic and embolization steps have been reported before $(11,12,19,20)$. Patients were either treated with selective (lobar or segmental) or super-selective injections.

CT scan. Multidetector CT images were acquired on a 64-slice CT scanner (Somatom Sensation 64, Siemens Medical Solutions, Erlangen, Germany) approximately $24 \mathrm{~h}$ after the cTACE procedure and prior to discharge. Only non-contrast CT scans were obtained based on the study standard abdomen protocol using the following parameters: $120 \mathrm{kVp}$ acquisitions, $545 \mathrm{~mA}$, scan speed, 0.33 $\mathrm{s} /$ revolution; detector collimation, $0.6 \mathrm{~mm} / \mathrm{row}$; helical pitch factor, $0.575 /$ revolution. After that, image reconstruction was performed using body kernel B30f, with a field of view of $400 \times 400 \times 220 \mathrm{~mm}$ (matrix size of $512 \times 512 \times 300$ ) with a voxel size of $0.78 \mathrm{~mm}^{3}$.

MRI. MRI of the abdomen was obtained using a standardized MRI protocol for liver masses in all patients on a 1.5-Tesla scanner (Siemens Magnetom Avanto; Siemens, Erlangen, Germany) with a phased array torso coil (repetition time $5.77 \mathrm{~ms} /$ echo time $2.77 \mathrm{~ms}$; field of view 320-400 mm; matrix, $192 \times 160$; slice thickness, 2.5 $\mathrm{mm}$; receiver bandwidth, $64 \mathrm{kHz}$; flip angle, $10^{\circ}$ ). The protocol included single-shot breath-hold gradient-echo diffusion-weighted echo-planar images in the axial dimension, axial T2-weighted fast spin-echo images, and unenhanced and contrast-enhanced $(0.1$ $\mathrm{mmol} / \mathrm{kg}$ intravenous gadopentetate; Magnevist; Bayer, Wayne, NJ, USA) breath-hold axial T1-weighted 3D fat-suppressed soiled gradient-echo images in the hepatic arterial phase $(20 \mathrm{~s}$ after contrast injection), portal venous phase (70 s after contrast injection) and delayed phase (180 s after contrast injection). 
A

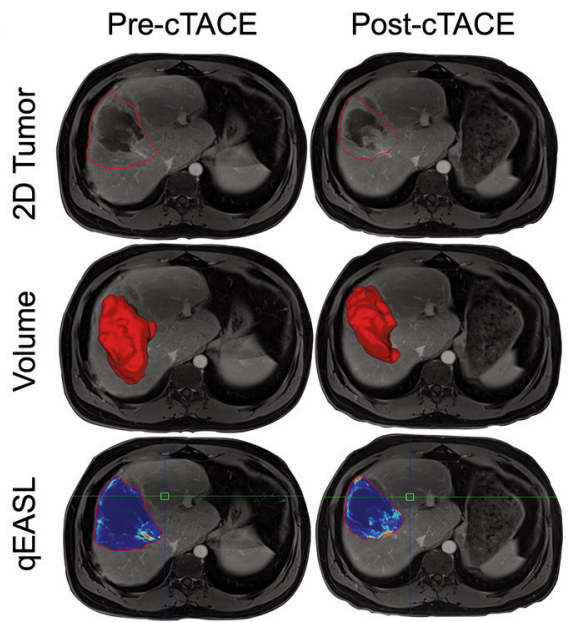

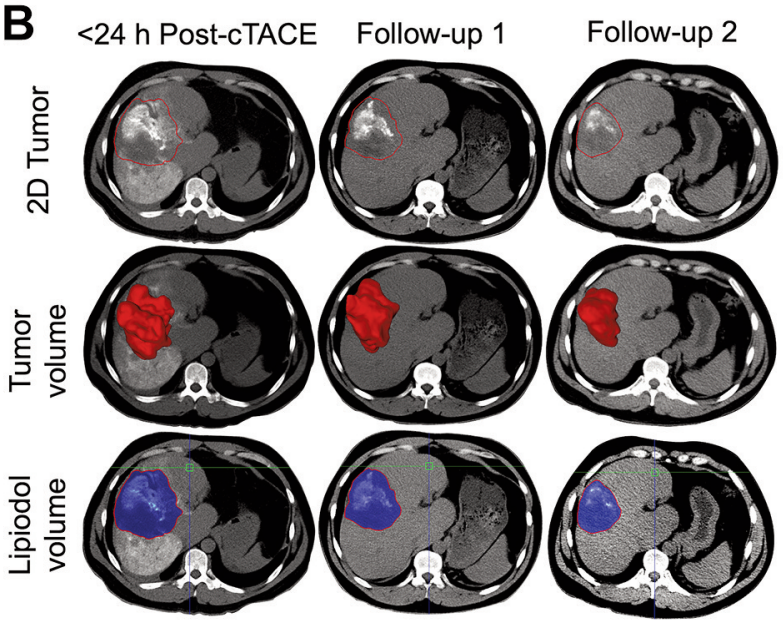

Figure 2. A: Magnetic resonance imaging (MRI), segmentation of liver tumors and analysis of quantitative $3 D$ European Association for the Study of the Liver metrics (qEASL). This figure demonstrates $2 D$ and volumetric tumor segmentation and qEASL evaluation of the volume of tumor enhancement prior to conventional trans-arterial chemoembolization (cTACE) and in follow-up MRI studies. B: Computed tomographic imaging, and lipiodol deposition segmentation, and qEASL analysis. This figure demonstrates $2 D$ and volumetric tumor segmentation and qEASL evaluation of lipiodol deposition pre cTACE and in follow-up computed tomography.

Image analysis. Measurements according to the response evaluation criteria in solid tumors (RECIST) and modified response evaluation criteria in solid tumors (mRECIST) metrics (21), as well as volumetric segmentation of the liver and targeted tumor, were carried out by a radiology resident (N.N., with 4.5 years of experience in abdominal MRI). All measurements were conducted using standard electronic calipers using Digital Imaging in Communications and Medicine files. To measure the diameter and area of tumor contrast enhancement, the reader re-evaluated the images in their different phases to distinguish the true extent of tumor burden. For 1D measurements, the two largest enhanced lesions were assessed. The arterial phase was used for NET, the venous phase was evaluated for CRC and the delayed phase for ICC.

A prototype $3 \mathrm{D}$ quantitative semiautomatic tumor analysis software (Medisys; Philips Research, Suresnes, France) was used for volumetric tumor assessment (9). 3D Segmentation masks of the tumors were created by three readers (I.R., J.M.M.v.B., and M.A.M.) each with 1 year of experience using the 3D tumor analysis program. The readers were supervised by a radiologist (N.N.). The area within the segmented mask was considered the total tumor volume measured in cubic centimeters. The volume of tumor enhancement $\left(\mathrm{cm}^{3}\right)$ was calculated by qEASL calculation, which has been described before in detail (9). Briefly, axial non-contrast and corresponding contrast-enhanced phases of MRI T1-weighted images were uploaded to the software. After subtraction, feeding the 3D tumor segmentation mask of the tumors, and placing a region of interest within healthy liver parenchyma, the software generated a color map of the enhanced tissue within the segmented 3D tumor mask automatically, with not enhanced, i.e., necrotic, areas of the tumor being represented in blue and enhanced and thus viable parts of the tumor being represented in red (Figure 2A). The software also calculated the volume of tumor enhancement automatically.

A decrease of $65 \%$ or more in the volume of tumor enhancement on MRI was considered a response to cTACE (22). The same segmentation method was used to determine lipiodol deposition $\left(\mathrm{cm}^{3}\right)$ using CT images (Figure 2B). Here, radiopaque lipiodol was represented by hyperdense voxels, in contrast to the previously mentioned MRI scans where hyperintense voxels represented the inflow of the contrast agent (i.e., viable tissue).

The following formula was used for evaluation of lipiodol washout on non-contrast $\mathrm{CT}$, and the result was calculated as the average change in lipiodol volume $\left(\mathrm{cm}^{3}\right)$ per day given by (i.e., the slope) (2):

\section{$\lambda=\Delta \mathrm{V} / \Delta \mathrm{t}$}

where was the change in tumor lipiodol volume between the first (within $24 \mathrm{~h}$ of cTACE) and follow-up non-contrast CT, and was the follow-up time in days.

Statistical analysis. Statistical analysis and data management was performed to find the most significant cutoff values for tumor size and enhancing tumor volume while looking into the lipiodol deposition and washout. For comparison of groups, the Mann-Whitney $U$-test and Kruskal-Wallis tests were used, the former for pairwise comparisons, the latter for multiple comparisons. Additionally, non-parametric bootstrapped exponential regression was used to evaluate the existence of an exponential washout rate over time over various strata. Lastly, a non-parametric bootstrap was used to evaluate the Pearson correlation between lipiodol deposition and tumor volume. For both bootstrap estimates, 100,000 bootstrap samples were conducted. Bootstrapping was employed to overcome the small sample size and allow for valid asymptotic estimation of the parameters. Throughout, a $p$-value of less than 0.05 was considered statistically significant.

\section{Results}

Study population characteristics. The studied patients were a typical cohort population (Table I) with a median overall survival of 10 months and a median number of cTACE treatments of two sessions per patient. Six (25\%) patients 
Table I. Demographic characteristics of the studied population.

\begin{tabular}{llc}
\hline Variable & & Value \\
\hline Gender, n (\%) & Male/female & $14 / 10(58.3 \% / 41.7 \%)$ \\
Age & Mean \pm SD & $59.63 \pm 14.36$ \\
Ethnicity, n (\%) & White & $20(83.3 \%)$ \\
& African American & $1(4.2 \%)$ \\
Median overall survival & Asian & $3(12.5 \%)$ \\
ECOG & Months, range & $10.00(3-81)$ \\
& 0 & 18 \\
Number of cTACE & 1 & 6 \\
Laboratory findings & Median (range) & $2(1-6)$ \\
& Serum bilirubin, mg/dl & $0.65 \pm 0.37$ \\
Ascites, n (\%) & Serum bilirubin, mg/dl & $3.93 \pm 0.49$ \\
Encephalopathy, $\mathrm{n}(\%)$ & Yes & $1.00 \pm 0.08$ \\
& Yes & $5(20.8 \%)$ \\
\hline
\end{tabular}

cTACE: Conventional trans-arterial chemoembolization; ECOG: Eastern Cooperative Oncology Group; INR: international normal ratio; SD: standard deviation.

had ICC, four (16.67\%) patients had NET and 14 (58.33\%) patients had CRC. Nine patients had a single lesion, while 15 patients had multiple hepatic lesions. While single target lesions were analyzed in all patients with ICC and NET, two different target lesions were analyzed in three patients with CRC; in total, six (22.22\%) ICC, four (14.82\%) NET and 17 $(62.96 \%)$ CRC target lesions were evaluated.

Baseline liver, tumor and lipiodol measurements. Table II demonstrates the baseline liver volume, largest tumor diameter, largest diameter of tumor enhancement, tumor volume, volume of contrasted-enhanced tumor based on tumor types and post cTACE response. Lipiodol volume within the tumor and the percentage of coverage is also shown in Table II, based on tumor type and post cTACE response.

Lipiodol washout. There was a negative exponential relationship between the lipiodol washout rate and the time interval between the initial and follow-up lipiodol imaging $(p<0.0001$; Figure 3$)$ for all tumors. This exponential curve showed lipiodol washout to be a time-dependent process with an estimated half-life of 23.09 days [95\% confidence intervaI $(\mathrm{CI})=20.34-25.85$ days] .

Lipiodol deposition and washout based on tumor type. No significant differences were identified in the volume of lipiodol deposition between NET, CRC, and ICC ( $p=0.96$; Figure 4A). However, the lipiodol washout rate was different between different types of tumors $(p<0.001$; Figure 4B-D). No exponential relationship with respect to time was observed in the washout rate among ICC tumors $(p=0.83)$. However, an exponential relationship was observed for CRC

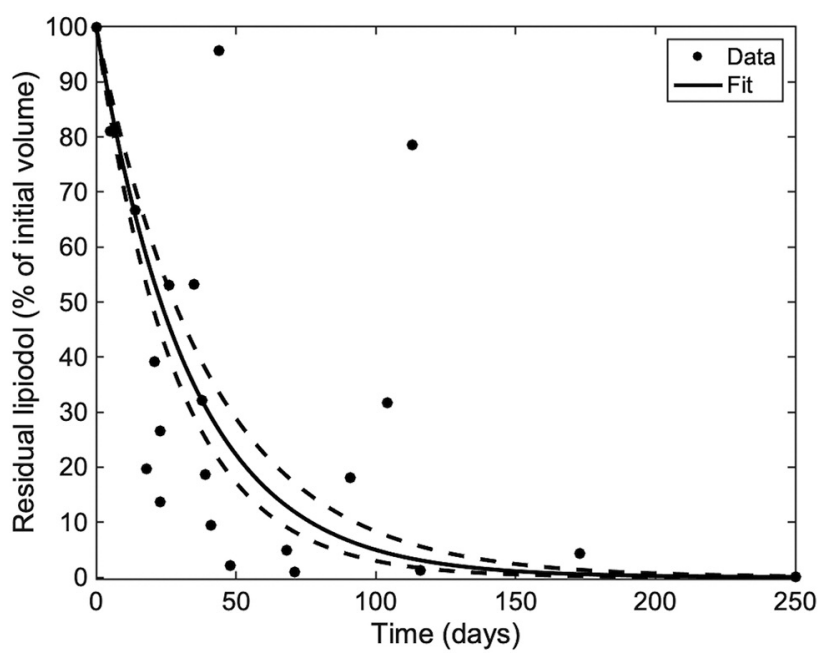

Figure 3. Tumor lipiodol washout for the entire cohort. This exponential curve shows lipiodol washout is a time-dependent process, with $R^{2}$ of 0.78. Dotted lines indicate the $95 \%$ confidence interval of the fitted line.

$(p<0.0001)$ and NET $(p<0.001)$. The lipiodol washout rate was slower in NET than in CRC, with half-lives for lipiodol washout of 54.61 days (95\% CI=42.63-75.97 days) and 19.39 days $(95 \% \mathrm{CI}=17.56-21.64$ days), respectively.

Lipiodol deposition and washout based on tumor size. There was a direct linear correlation between lipiodol deposition and tumor size (Pearson correlation $\mathrm{r}=0.63, p<0.01$ ). The tumor volume of $300 \mathrm{~cm}^{3}$ was determined as the most significant cutoff value to differentiate for lipiodol deposition based on tumor size. Lipiodol deposition was significantly higher in tumors larger than $300 \mathrm{~cm}^{3}(p=0.031$; Figure 5A). In both groups $\left(\geq 300 \mathrm{~cm}^{3}\right.$ and $\left.<300 \mathrm{~cm}^{3}\right)$, a negative exponential relationship was observed over time for the lipiodol washout rate $(p<0.00001$, Figure 5B; and $p<0.01$, Figure 5C, respectively). In patients with tumors $300 \mathrm{~cm}^{3}$ or larger, the lipiodol washout half-life was 25.43 days (95\% $\mathrm{CI}=18.32-41.54$ days), while it was 22.71 days $(95 \%$ $\mathrm{CI}=20.27-25.80)$ in smaller tumors .

Lipiodol deposition and washout based on tumor enhancement burden. Based on receiver operating characteristics analysis, a tumor enhancement burden of $60 \%$ was determined as the most significant cutoff value to detect differences in lipiodol deposition. Lipiodol deposition was moderately higher in tumors with tumor enhancement burden of $60 \%$ or higher than when compared to tumors with lower tumor enhancement burden ( $p=0.067$, Figure 6A).

In both groups (tumor enhancement burden $\geq 60 \%$ and $<60 \%$ ), a negative exponential relationship was observed over time in lipiodol washout $(p<0.01$; Figure $6 \mathrm{~B})$ and $(p<0.0001$; Figure 6C), respectively. In particular, in patients 
Table II. Baseline liver volume, tumor and lipiodol measurements (mean $\pm S D$ ).

\begin{tabular}{|c|c|c|c|c|c|c|}
\hline \multirow[t]{2}{*}{ Variable } & \multirow[t]{2}{*}{ Total } & \multicolumn{2}{|c|}{ Tumor type } & \multirow[b]{2}{*}{$\mathrm{CRC}$} & \multicolumn{2}{|c|}{ Response status } \\
\hline & & ICC & NET & & Response & No response \\
\hline Liver volume, $\mathrm{cm}^{3}$ & $2,268.9 \pm 923.62$ & $2,234.0 \pm 961.9$ & $2,946.5 \pm 3.5$ & $2,173.5 \pm 979.2$ & $2,427.3 \pm 1,550.2$ & $2,229.4 \pm 769.2$ \\
\hline Tumor largest diameter, $\mathrm{cm}$ & $12.6 \pm 4.0$ & $13.0 \pm 5.1$ & $13.2 \pm 1.5$ & $12.4 \pm 3.9$ & $10.0 \pm 4.0$ & $12.8 \pm 3.6$ \\
\hline $\begin{array}{l}\text { Enhancement, largest } \\
\text { diameter, cm }\end{array}$ & $7.0 \pm 1.9$ & $7.1 \pm 2.6$ & $8.9 \pm 1.4$ & $6.7 \pm 1.5$ & $5.4 \pm 2.6$ & $7.4 \pm 1.4$ \\
\hline Tumor volume, $\mathrm{cm}^{3}$ & $751.6 \pm 770.0$ & $1,047.5 \pm 975.2$ & $1,349.3 \pm 529.8$ & $553.6 \pm 664.4$ & $772.1 \pm 1,139.3$ & $779.3 \pm 715.9$ \\
\hline Enhancement volume, $\mathrm{cm}^{3}$ & $248.7 \pm 254.0$ & $406.0 \pm 315.9$ & $666.5 \pm 45.1$ & $130.2 \pm 122.6$ & $86.4 \pm 77.4$ & $309.7 \pm 280.8$ \\
\hline Tumor lipiodol volume, $\mathrm{cm}^{3}$ & $235.9 \pm 211.0$ & $198.4 \pm 112.6$ & $215.0 \pm 223.9$ & $254.2 \pm 240.8$ & $228.1 \pm 263.1$ & $246.5 \pm 214.4$ \\
\hline Tumor lipiodol coverage, $\%$ & $49.8 \pm 23.4$ & $43.9 \pm 18.9$ & $50.3 \pm 44.4$ & $51.7 \pm 19.9$ & $41.6 \pm 20.5$ & $48.5 \pm 18.0$ \\
\hline
\end{tabular}

CRC: Colorectal carcinoma; ICC: intrahepatic cholangiocarcinoma; NET: neuroendocrine tumor; SD: standard deviation.

A

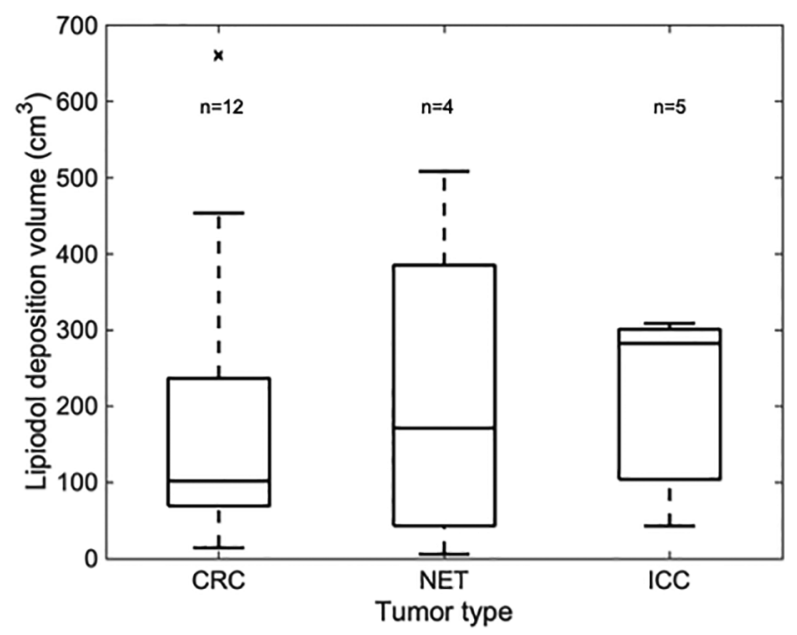

C

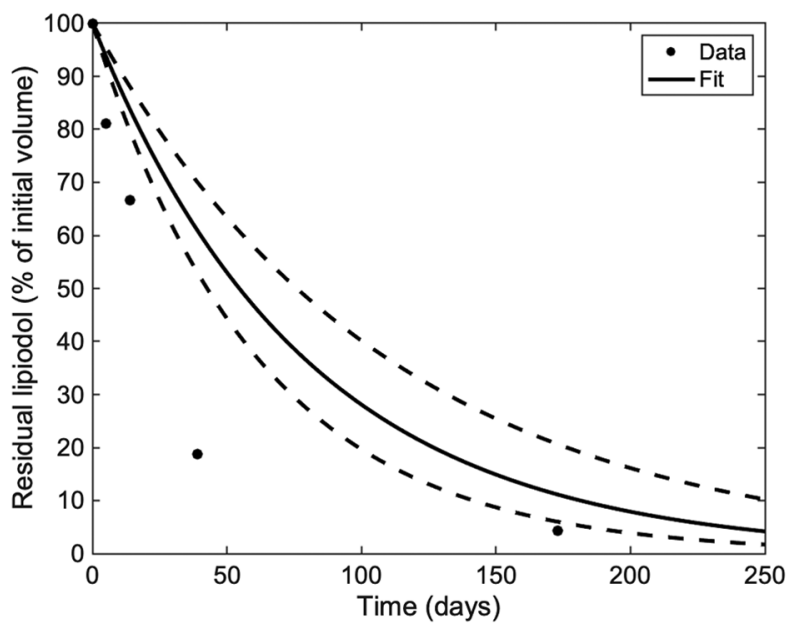

B

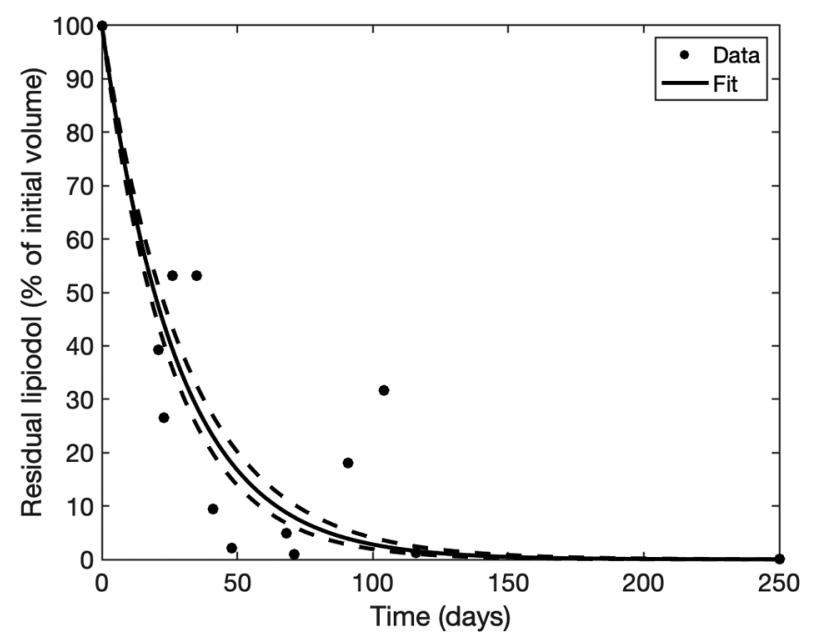

D

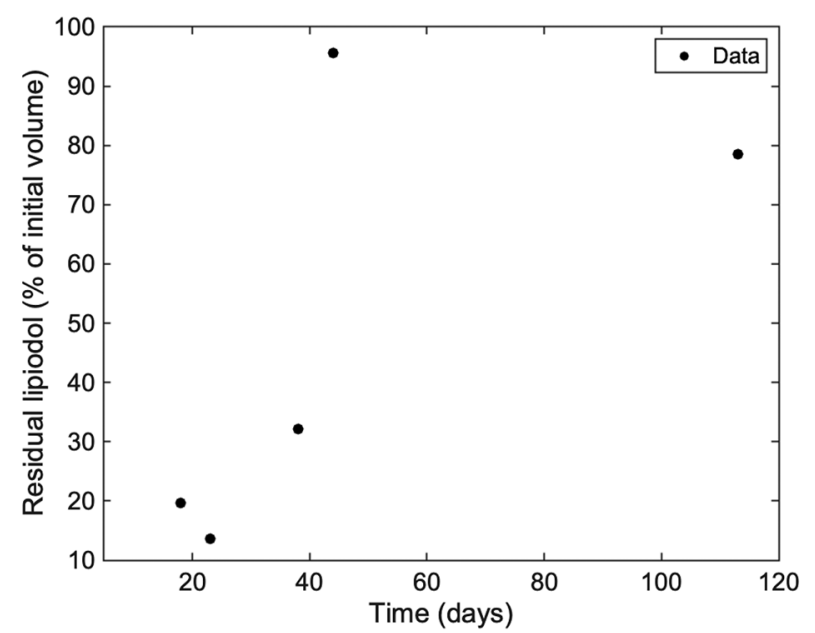

Figure 4. Tumor lipiodol deposition and washout based on tumor type. A: Lipiodol deposition was not different between colorectal cancer (CRC), intrahepatic cholangiocarcinoma (ICC), and neuroendocrine tumor (NET). The lipiodol washout curves for CRC (B), NET (C), and ICC (D) had $R^{2}$ of 0.8985, 0.8909, and 0.0322, respectively. Dotted lines indicate the $95 \%$ confidence interval of the fitted line. No fit is provided for ICC given the absence of an exponential relationship. 

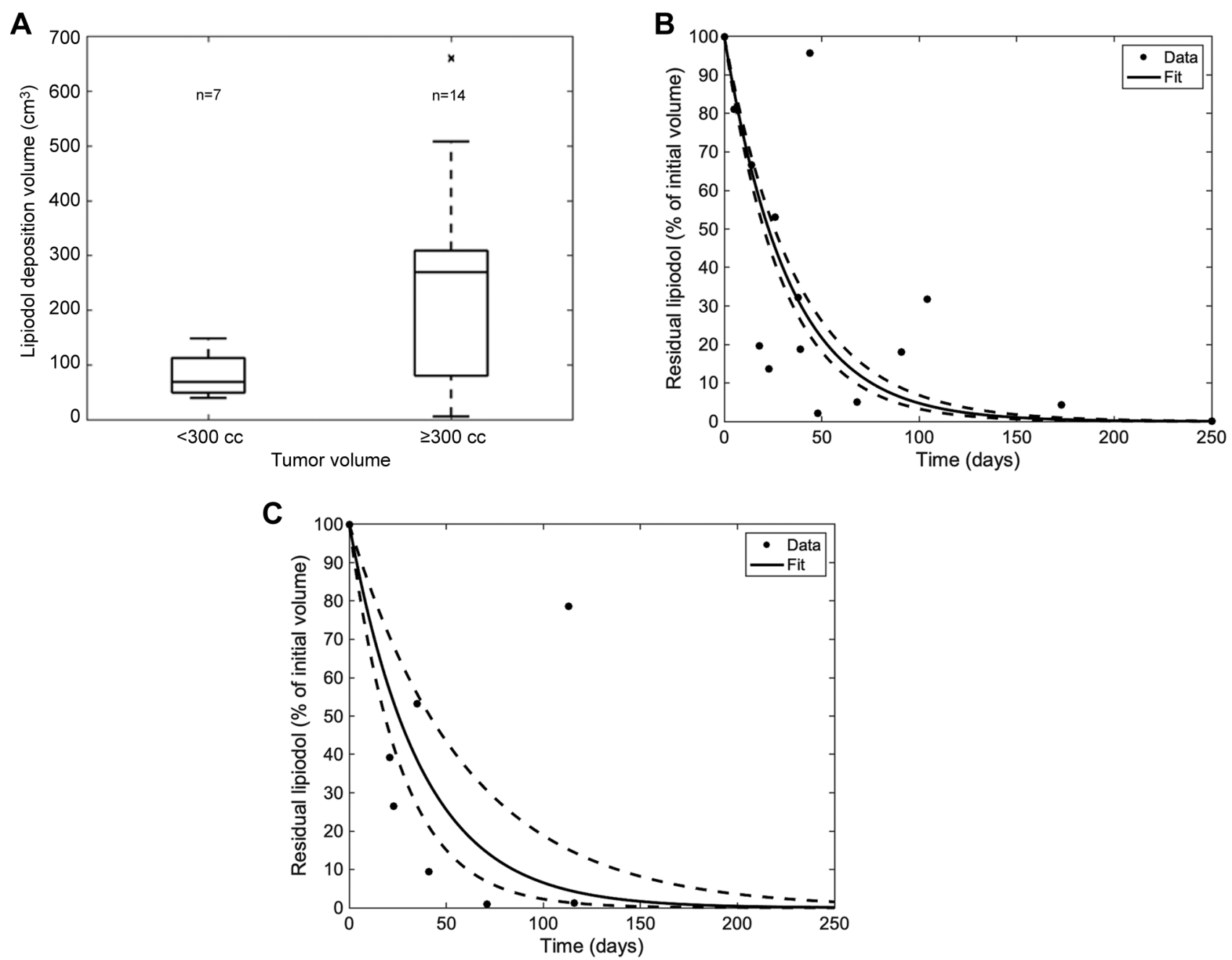

Figure 5. Lipiodol deposition and washout based on tumor volume. A: The lipiodol deposition was greater in tumors larger than $300 \mathrm{~cm}^{3}$. Using a tumor volume cut-off of $300 \mathrm{~cm}^{3}$ patients were divided into groups with $\geq 300 \mathrm{~cm}^{3}(B)$ and $<300 \mathrm{~cm}^{3}(C)$, with $R^{2}$ of 0.8496 and 0.5731 , respectively. Dotted lines indicate the $95 \%$ confidence interval of the fitted line.

with $60 \%$ or more tumor enhancement burden, the lipiodol washout half-life was 54.76 days (95\% CI=34.76-130.18 days), while lipiodol washout half-life was 29.45 (95\% $\mathrm{CI}=26.03-33.92$ days) in patients with less than $60 \%$ tumor enhancement burden. Furthermore, the absence of an overlap between CIs for the two groups suggests that patients with a tumor enhancement burden of greater than $60 \%$ had a statistically higher washout half-life.

Lipiodol washout and response. Based on both mRECIST and qEASL criteria, $5(22.7 \%)$ target lesions showed response to cTACE.

The lipiodol deposition in responders was not significantly higher than the deposition in non-responders $(p=0.19$; Figure 7A). There was no statistically significant exponential fit for the responders ( $p=0.20$; Figure $7 \mathrm{~B})$. In contrast, among the non-responders, a statistically significant negative exponential washout rate was observed $(p<0.00001$; Figure 7C). Correspondingly, the lipiodol half-life in non-responders was found to be 34.42 days (95\% CI=28.30-43.93 days).

\section{Discussion}

Our findings showed that lipiodol washout is a timedependent process which proceeds in a negative exponential pattern, i.e., a faster initial washout phase followed by a later slower washout phase. Lipiodol is an oil-based contrast medium composed of fatty acid ethylic esters conjugated with iodine (37-48\% concentration) (23). The mechanism of selective deposition of lipiodol inside hepatic primary and 
A

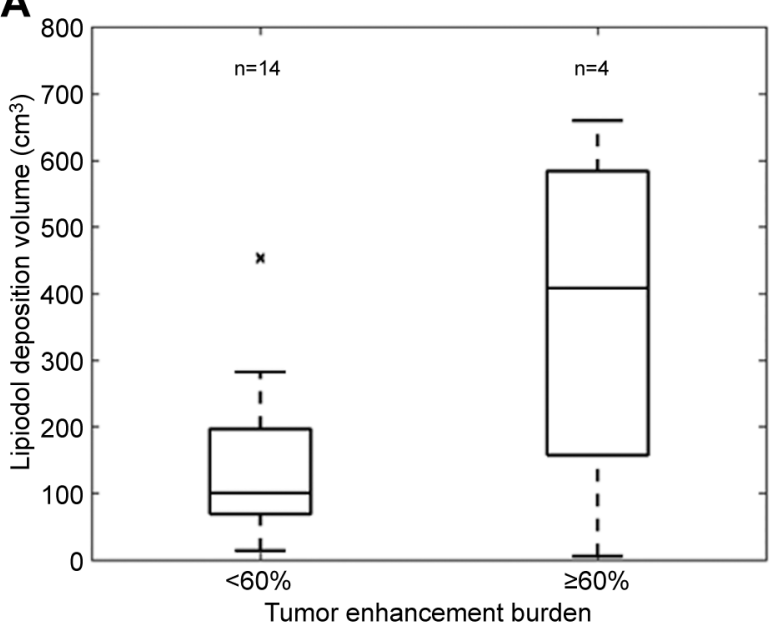

B

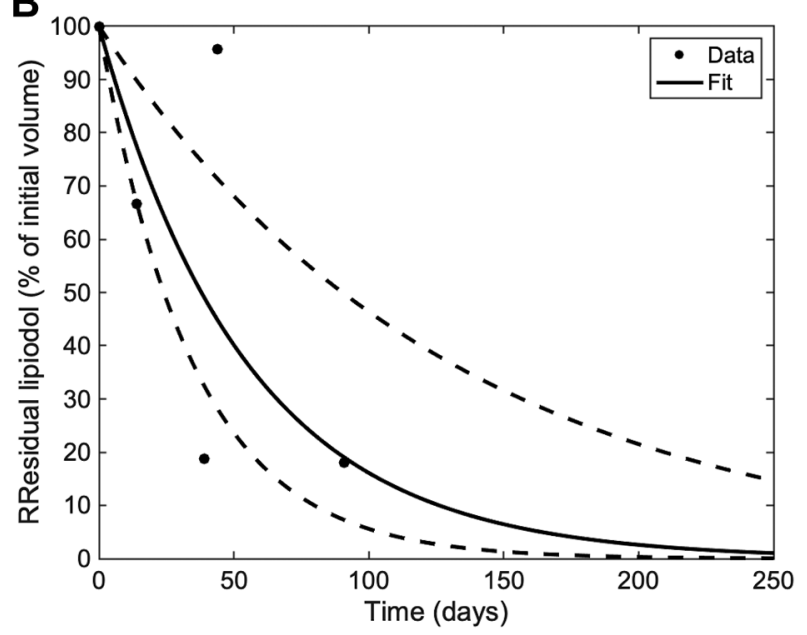

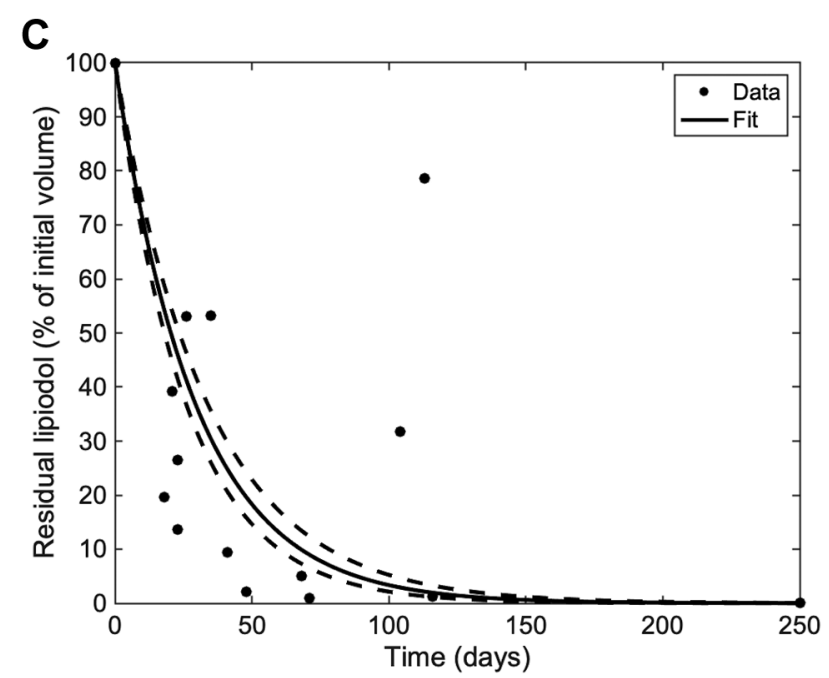

Figure 6. Tumor lipiodol deposition and washout based on the burden of tumor enhancement. A: Tumor lipiodol deposition was higher in tumors with an enhancement burden of $60 \%$ or greater. Lipiodol washout based on tumor enhancement burden of $\geq 60 \%(B)$ and $<60 \%(C)$ had $R^{2}$ of 0.8283 and 0.7459 , respectively. Dotted lines indicate the $95 \%$ confidence interval of the fitted line.

metastatic tumors is only partially known. Hepatic tumors have a different degree of vascularization based on their nature and self-promoting angiogenesis. These tumors are usually vascularized with arterial supply mediated by the secretion of factors such as vascular endothelial growth factor. Tumor arterial supply dominates over portal venous supply with progressive involution creating a 'siphon effect'. This increased tumor neo-vascularity has an affinity for lipiodol $(24,25)$. Furthermore, arterial vessels produced through tumor neo-angiogenesis are tortuous and irregular, with their caliber moderately increased and segments lacking tunica muscularis. This anomalous neo-angiogenesis allows leakage of contrast into the perivascular, intercellular space (25). Lastly, during neoplastic transformation, the plasma membrane may undergo a biochemical modification, rendering it more lipophilic; this would favor adhesion of lipiodol to the membrane and its subsequent endocytosis into neoplastic cells (26). A combination of these changes results in varying degrees of lipiodol deposition and washout within the tumor, which is best imaged on CT scans post cTACE.

Lipiodol washout from embolized lesions occurs slowly because of insufficient portal vascularization (the main mechanism responsible for non-neoplastic washout), absence of reticuloendothelial cells such as Kupffer cells, and lack of lymphatic vessels $(5,27)$. These histopathological characteristics are different in various tumor types. Our findings showed that the rate of lipiodol washout followed a negative exponential curve, i.e., a faster initial phase followed by a later slower phase. In addition, we demonstrated that the lipiodol washout rate was fastest in $\mathrm{CRCs}$ as compared to 
A

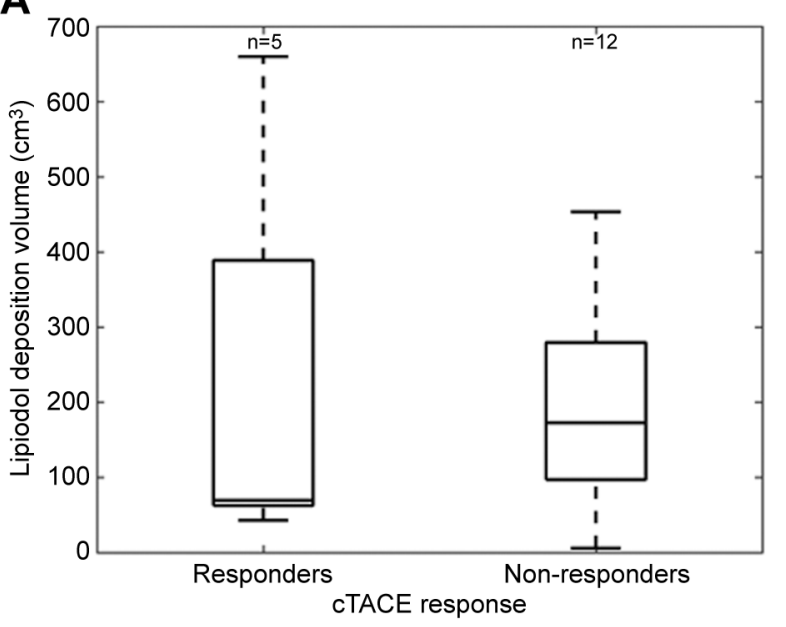

B

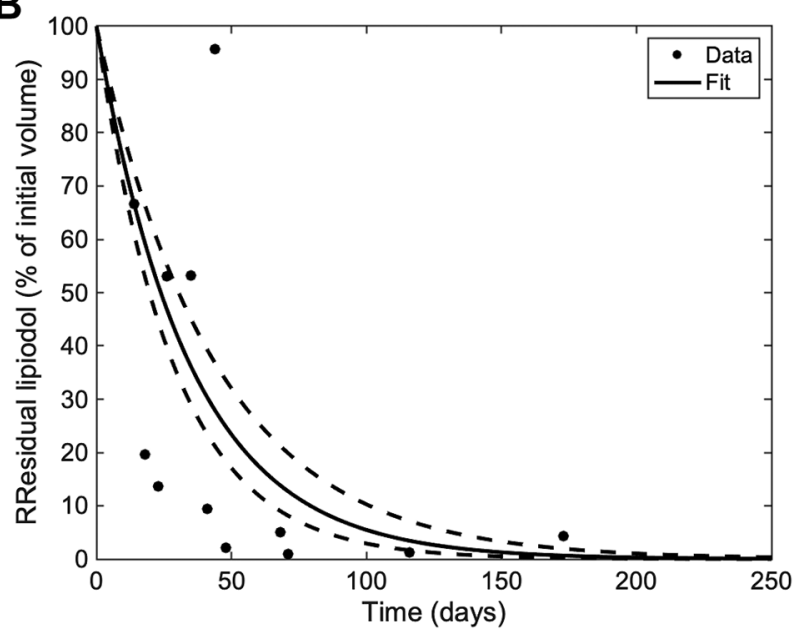

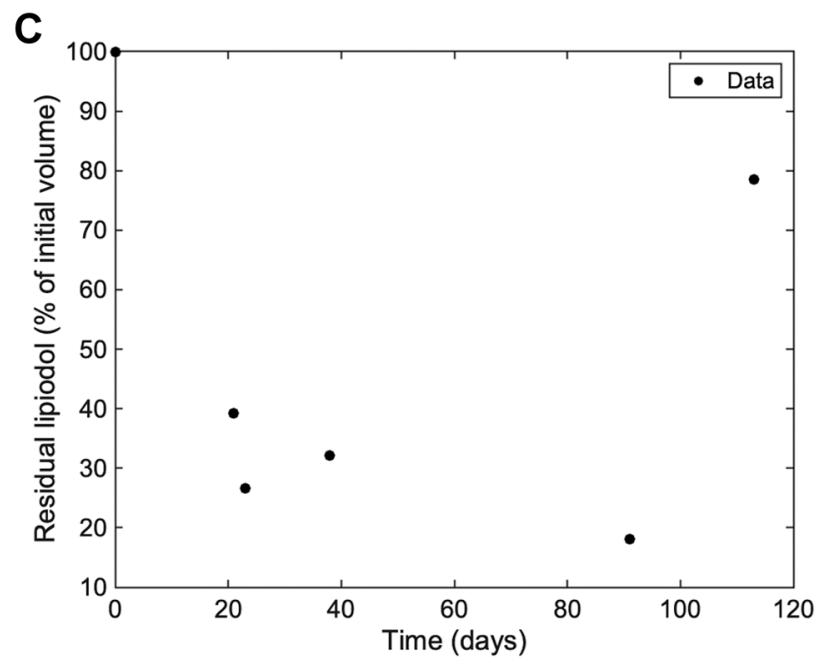

Figure 7. Tumor lipiodol washout rate for the entire cohort based on response to conventional trans-arterial chemoembolization (cTACE). A: Lipiodol deposition was not significantly different between the responders and non-responders. Tumor lipiodol washout rate for the entire cohort for (B) responders and $(C)$ non-responders, with $R^{2}$ of 0.3792 and 0.6866 , respectively. Dotted lines indicate the $95 \%$ confidence interval of the fitted line. No fit is provided for non-responders given the absence of an exponential relationship.

NETs and ICCs. In fact, we did not find clear lipiodol washout pattern for ICCs, which is likely related to the fibrotic nature of ICC tumor. The pattern of washout across the different types of tumors may be related to unique histopathological characteristics of ICCs, NETs, and CRCs, including the extent of fibrotic tissue, vascularity, lymphatic vessels, Kupffer and phagocyting cell density (28-31), each of which can facilitate or slow lipiodol washout and clearance from the tumor.

Our findings also showed that the lipiodol washout rate was slower in tumors with a tumor enhancement burden of $60 \%$ or greater. The burden of tumor enhancement reflects the vascularized part of the tumor with anomalous neoangiogenesis that favors leakage of lipiodol into the intercellular space $(24,25)$ and adhesion of lipiodol to the tumor cell membrane with its subsequent endocytosis into neoplastic cells (26). This might be related to the speed at which the tumor microenvironment can clear lipiodol. The larger the volume of tumor enhancement, the more lipiodol can be deposited during cTACE and the more lipiodol to be cleared.

Our findings indicated that there was not a statistically significant lipiodol washout in responders to cTACE; however, non-responders did experience some degree of washout. Absence of washout in responders is suggestive of successful trans-arterial delivery of lipiodol, resulting in embolization of arterial and portal vessels supplying the tumor, tumor cell necrosis, and lipiodol retention within the tumor $(32,33)$. This should be an indirect indicator of efficient delivery of chemotherapeutic agents (33), better induction of ischemia and necrosis, and consequently prolonged lipiodol retention and slower washout. 
Although tumors with a volume of $300 \mathrm{~cm}^{3}$ or larger than had more lipiodol deposition, the lipiodol washout rate was slightly faster. Since only a predetermined volume of lipiodol $(10 \mathrm{ml})$ is commonly used for cTACE, faster lipiodol washout may suggest that the standard volume of lipiodol might be suboptimal for sufficient embolization of supplying arteries, leaving residual blood flow behind and resulting in faster lipiodol washout. It is expected that larger tumors would accommodate larger volumes of lipiodol, which was seen in our study in tumors with a volume of $300 \mathrm{~cm}^{3}$ or more. Studies have shown that larger ICCs, NETs and CRCs are more likely to have larger areas of central necrosis which cannot retain lipiodol (29-31). Therefore, the necrotic proportion and vascularity of the tumor should be considered while correcting for lipiodol deposition.

This study shows lipiodol deposition and washout rates in the three most common non-HCC types of hepatic lesions treated by cTACE. Furthermore, to our knowledge, this is the only study examining the difference in the rate of lipiodol washout between these three types of hepatic tumors. The study is limited by the small sample size, a limitation partially mitigated by the use of non-parametric methods and bootstrap statistical methods. Furthermore, given the small sample size, regression estimates were not adjusted for possible confounding, a consideration which ought to be accounted for in future studies. Additionally, although we used a volumetric method to analyze lipiodol deposition, we did not evaluate the homogeneity, density, coverage and pattern of lipiodol deposition in tumors.

In conclusion, lipiodol washout is a time-dependent, negative exponential process that varies in tumors with different histopathology, resulting in faster lipiodol washout rates in CRCs than NETs. Lipiodol washout occurs faster in patients with tumors smaller than $300 \mathrm{~cm}^{3}$ and tumor enhancement burden of less than $60 \%$. When lipiodol deposition is used to predict the response to cTACE, tumor types and features should be considered as factors which potentially influence lipiodol washout and deposition.

\section{Conflicts of Interest}

JC reports grant support from the Society of Interventional Oncology, Guerbet Pharmaceuticals, Philips Healthcare, Boston Scientific, Yale Center for Clinical Investigation, and the NIH R01CA206180 outside the submitted work. M.L. is a current employee and stockholder of Visage Imaging, Inc. and former Philips Research North America employee, and has grant support from NIH R01CA206180. All other Authors declare no conflicts of interest.

\section{Authors' Contributions}

Nariman Nezami: Concept, qualitative and quantitative analyzes, drafting, review and revision. Johanna Maria Mijntje van Breugel: Concept, and review. Menelaos Konstantinidis: Statistical analysis. Julius Chapiro: Concept and review. Lynn Jeanette Savic: Review and revision. Milena A. Miszczuk: Quantitative analysis and review.
Irvin Rexha: Quantitative analysis and review. MingDe Lin: supervised and reviewed. Kelvin Hong: supervised and reviewed. Christos Georgiades: Supervision and revision.

\section{References}

1 Liapi E and Geschwind JF: Transcatheter arterial chemoembolization for liver cancer: is it time to distinguish conventional from drug-eluting chemoembolization? Cardiovasc Intervent Radiol 34(1): 37-49, 2011. PMID: 21069333. DOI: 10.1007/s00270-010-0012-y

2 Lencioni R, Petruzzi P and Crocetti L: Chemoembolization of hepatocellular carcinoma. Semin Intervent Radiol 30(1): 3-11, 2013. PMID: 24436512. DOI: 10.1055/s-0033-1333648

3 van Breugel JMM, Geschwind JF, Mirpour S, Savic LJ, Zhang X, Duran R, Lin M, Miszczuk M, Liapi E and Chapiro J: Theranostic application of lipiodol for transarterial chemoembolization in a VX2 rabbit liver tumor model. Theranostics 9(13): 3674-3686, 2019. PMID: 31281506 . DOI: 10.7150/thno.32943

4 Nakakuma K, Tashiro S, Hiraoka T, Uemura K, Konno T, Miyauchi Y and Yokoyama I: Studies on anticancer treatment with an oily anticancer drug injected into the ligated feeding hepatic artery for liver cancer. Cancer 52(12): 2193-2200, 1983. PMID: 6196102. DOI: 10.1002/1097-0142(19831215)52: $12<2193$ ::aid-cncr2820521203>3 .0.co;2-r

5 Shin SW: The current practice of transarterial chemoembolization for the treatment of hepatocellular carcinoma. Korean J Radiol 10(5): 425-434, 2009. PMID: 19721826. DOI: 10.3348/kjr.2009.10.5.425

6 Imaeda T, Yamawaki Y, Seki M, Goto H, Inuma G, Kanematsu M, Mochizuki R, Doi H, Saji S and Shimokawa K: Lipiodol retention and massive necrosis after lipiodol-chemoembolization of hepatocellular carcinoma: correlation between computed tomography and histopathology. Cardiovasc Intervent Radiol 16(4): 209-213, 1993. PMID: 8402781. DOI: 10.1007/BF02602962

7 Hu J, Maybody M, Cao G, Wang X, Chen H, Zhu X, Yang R and Wang $\mathrm{X}$ : Lipiodol retention pattern assessed by cone beam computed tomography during conventional transarterial chemoembolization of hepatocellular carcinoma: accuracy and correlation with response. Cancer Imaging 16(1): 32, 2016. PMID: 27716376. DOI: 10.1186/s40644-016-0090-4

8 Chen R, Geschwind JF, Wang Z, Tacher V and Lin M: Quantitative assessment of lipiodol deposition after chemoembolization: comparison between cone-beam CT and multidetector CT. J Vasc Interv Radiol 24(12): 1837-1844, 2013. PMID: 24094672. DOI: 10.1016/j.jvir.2013.08.017

9 Lin M, Pellerin O, Bhagat N, Rao PP, Loffroy R, Ardon R, Mory B, Reyes DK and Geschwind JF: Quantitative and volumetric European Association for the Study of the Liver and Response Evaluation Criteria in Solid Tumors measurements: feasibility of a semiautomated software method to assess tumor response after transcatheter arterial chemoembolization. J Vasc Interv Radiol 23(12): 1629-1637, 2012. PMID: 23177109. DOI: 10.1016/j.jvir. 2012.08.028

10 Chapiro J, Lin M, Duran R, Schernthaner RE and Geschwind JF: Assessing tumor response after loco-regional liver cancer therapies: the role of 3D MRI. Expert Rev Anticancer Ther 15(2): 199-205, 2015. PMID: 25371052. DOI: 10.1586/14737140.2015.978861

11 Tacher V, Lin M, Duran R, Yarmohammadi H, Lee H, Chapiro J, Chao M, Wang Z, Frangakis C, Sohn JH, Maltenfort MG, Pawlik T and Geschwind JF: Comparison of existing response 
criteria in patients with hepatocellular carcinoma treated with transarterial chemoembolization using a 3D quantitative approach. Radiology 278(1): 275-284, 2016. PMID: 26131913. DOI: $10.1148 /$ radiol.2015142951

12 Sahu S, Schernthaner R, Ardon R, Chapiro J, Zhao Y, Sohn JH, Fleckenstein F, Lin M, Geschwind JF and Duran R: Imaging biomarkers of tumor response in neuroendocrine liver metastases treated with transarterial chemoembolization: can enhancing tumor burden of the whole liver help predict patient survival? Radiology 283(3): 883-894, 2017. PMID: 27831830. DOI: 10.1148/radiol.2016160838

13 Ghani MA, Fereydooni A, Chen E, Letzen B, Laage-Gaupp F, Nezami N, Deng Y, Gan G, Thakur V, Lin M, Papademetris X, Schernthaner RE, Huber S, Chapiro J, Hong K and Georgiades $\mathrm{C}$ : Identifying enhancement-based staging markers on baseline MRI in patients with colorectal cancer liver metastases undergoing intra-arterial tumor therapy. Eur Radiol, 2021. PMID: 34061209. DOI: 10.1007/s00330-021-08058-7

14 Rexha I, Laage-Gaupp F, Chapiro J, Miszczuk MA, van Breugel JMM, Lin M, Konstantinidis M, Duran R, Gebauer B, Georgiades C, Hong K and Nezami N: Role of 3D quantitative tumor analysis for predicting overall survival after conventional chemoembolization of intrahepatic cholangiocarcinoma. Sci Rep 11(1): 9337, 2021. PMID: 33927226. DOI: 10.1038/s41598-021-88426-x

15 Wang Z, Chen R, Duran R, Zhao Y, Yenokyan G, Chapiro J, Schernthaner R, Radaelli A, Lin M and Geschwind JF: Intraprocedural 3D quantification of lipiodol deposition on conebeam CT predicts tumor response after transarterial chemoembolization in patients with hepatocellular carcinoma. Cardiovasc Intervent Radiol 38(6): 1548-1556, 2015. PMID: 26001366. DOI: 10.1007/s00270-015-1129-9

16 Wang Z, Lin M, Lesage D, Chen R, Chapiro J, Gu T, Tacher V, Duran R and Geschwind JF: Three-dimensional evaluation of lipiodol retention in HCC after chemoembolization: a quantitative comparison between CBCT and MDCT. Acad Radiol 21(3): 393-399, 2014. PMID: 24507426. DOI: 10.1016/ j.acra.2013.11.006

17 Miszczuk MA, Chapiro J, Geschwind JH, Thakur V, Nezami N, Laage-Gaupp F, Kulon M, van Breugel JMM, Fereydooni A, Lin M, Savic LJ, Tegel B, Wahlin T, Funai E and Schlachter T: Lipiodol as an imaging biomarker of tumor response after conventional transarterial chemoembolization: Prospective clinical validation in patients with primary and secondary liver cancer. Transl Oncol 13(3): 100742, 2020. PMID: 32092672. DOI: $10.1016 /$ j.tranon.2020.01.003

18 Fronda M, Doriguzzi Breatta A, Gatti M, Calandri M, Maglia C, Bergamasco L, Righi D, Faletti R and Fonio P: Quantitative assessment of HCC wash-out on CT is a predictor of early complete response to TACE. Eur Radiol 31(9): 6578-6588, 2021. PMID: 33738601. DOI: 10.1007/s00330-021-07792-2

19 Chapiro J, Duran R, Lin M, Schernthaner R, Lesage D, Wang Z, Savic LJ and Geschwind JF: Early survival prediction after intraarterial therapies: a 3D quantitative MRI assessment of tumour response after TACE or radioembolization of colorectal cancer metastases to the liver. Eur Radiol 25(7): 1993-2003, 2015. PMID: 25636420. DOI: 10.1007/s00330-015-3595-5

20 Geschwind JF, Kudo M, Marrero JA, Venook AP, Chen XP, Bronowicki JP, Dagher L, Furuse J, Ladrón de Guevara L, Papandreou C, Sanyal AJ, Takayama T, Ye SL, Yoon SK, Nakajima $\mathrm{K}$, Lehr $\mathrm{R}$, Heldner $\mathrm{S}$ and Lencioni R: TACE treatment in patients with sorafenib-treated unresectable hepatocellular carcinoma in clinical practice: Final analysis of GIDEON. Radiology 279(2): 630-640, 2016. PMID: 26744927. DOI: $10.1148 /$ radiol.2015150667

21 Subbiah V, Chuang HH, Gambhire D and Kairemo K: Defining clinical response criteria and early response criteria for precision oncology: Current state-of-the-art and future perspectives. Diagnostics (Basel) 7(1): 10, 2017. PMID: 28212290. DOI: 10.3390/diagnostics7010010

22 Stroehl YW, Letzen BS, van Breugel JM, Geschwind JF and Chapiro J: Intra-arterial therapies for liver cancer: assessing tumor response. Expert Rev Anticancer Ther 17(2): 119-127, 2017. PMID: 27983883. DOI: 10.1080/14737140.2017.1273775

23 Cheng KT: Lipiodol-loaded poly(oxyethylene)-blockpoly(oxypropylene)-block-poly(oxyethylene) triblock copolymers/ polyethylene glycol-nanoparticles. 2008. PMID: 20641972.

24 Chen CS, Li FK, Guo CY, Xiao JC, Hu HT, Cheng HT, Zheng L, Zong DW, Ma JL, Jiang L and Li HL: Tumor vascularity and lipiodol deposition as early radiological markers for predicting risk of disease progression in patients with unresectable hepatocellular carcinoma after transarterial chemoembolization. Oncotarget 7(6): 7241-7252, 2016. PMID: 26769845. DOI: 10.18632 /oncotarget.6892

25 Kan Z: Dynamic study of iodized oil in the liver and blood supply to hepatic tumors. An experimental investigation in several animal species. Acta Radiol Suppl 408: 1-25, 1996. PMID: 9014948.

26 Becker S, Ardisson V, Lepareur N, Sergent O, Bayat S, Noiret N, Gaboriau F, Clément B, Boucher E, Raoul JL and Garin E: Increased Lipiodol uptake in hepatocellular carcinoma possibly due to increased membrane fluidity by dexamethasone and tamoxifen. Nucl Med Biol 37(7): 777-784, 2010. PMID: 20870152. DOI: 10.1016/j.nucmedbio.2010.03.013

27 Kan Z, McCuskey PA, Wright KC and Wallace S: Role of Kupffer cells in iodized oil embolization. Invest Radiol 29(11): 990-993, 1994. PMID: 7890514. DOI: 10.1097/00004424199411000-00007

28 Van Beers BE: Diagnosis of cholangiocarcinoma. HPB (Oxford) 10(2): 87-93, 2008. PMID: 18773062. DOI: 10.1080/ 13651820801992716

29 Patel T: Cholangiocarcinoma-controversies and challenges. Nat Rev Gastroenterol Hepatol 8(4): 189-200, 2011. PMID: 21460876. DOI: $10.1038 /$ nrgastro.2011.20

30 Lewis RB, Lattin GE Jr and Paal E: Pancreatic endocrine tumors: radiologic-clinicopathologic correlation. Radiographics 30(6): 1445-1464, 2010. PMID: 21071369. DOI: $10.1148 / \mathrm{rg} .306105523$

31 Shore PA: Actions of amfonelic acid and other non-amphetamine stimulants on the dopamine neuron. J Pharm Pharmacol 28(11): 855-857, 1976. PMID: 11304. DOI: 10.1111/j.2042-7158.1976. tb04078.x

32 Idée JM and Guiu B: Use of Lipiodol as a drug-delivery system for transcatheter arterial chemoembolization of hepatocellular carcinoma: a review. Crit Rev Oncol Hematol 88(3): 530-549, 2013. PMID: 23921081. DOI: 10.1016/j.critrevonc.2013.07.003

33 Kan Z and Madoff DC: Liver anatomy: microcirculation of the liver. Semin Intervent Radiol 25(2): 77-85, 2008. PMID: 21326548. DOI: $10.1055 / \mathrm{s}-2008-1076685$

Received July 5, 2021

Revised August 18, 2021

Accepted September 6, 2021 